\title{
Photochemically-induced fluorescence flow-through optosensors: concepts and applications
}

\begin{abstract}
Researchers are concerned, more than ever, about the need to develop analytical methods that present not only the usually required characteristics (such as sensitivity and selectivity) but also easy automation and, especially, environmental friendliness. The use of flow analysis has always proved to be a suitable methodology for handling solutions, hence performing methods related to wet chemical analysis. Different flow methodologies have been used, since the early flow injection analysis to the more recent multi commutated methodologies, each of them presenting special characteristics. In this review, we will focus on the implementation of Solid Phase Spectroscopy (SPS) in flow analysis. This coupling increases the selectivity and sensitivity of the analytical methods in a simple way. In addition, most of the analytical methods use UV-Vis spectroscopy and fluorescence detection techniques. However, these techniques are not suitable for some analytes, hence the potential use of Photo chemicallyInduced Fluorescence (PIF). In this review, we will cover the fundamental aspects of the coupling of SPS and flow analysis methods, which is called Flow-Through Opt sensing. We will pay attention to the concepts of each flow methodology, paying special attention to the use of PIF detection. We will discuss the advantages and disadvantages as well as the applications that have been carried out to date. Finally, we will provide our opinion about the future trends of this analytical approach.
\end{abstract}

Keywords: automation, flow injection analysis, sequential injection analysis, multicommutation, multipumping, sensor, pesticide, contaminant, flow analysis, friendliness, spectroscopy, environment
Volume I Issue 2 - 2017

\author{
Llorent Martinez EJ,' Molina Garcia L² \\ Department of Physical and Analytical Chemistry, University of \\ Jaen, Spain
}

Correspondence: Eulogio Jose Llorent Martinez, Department of Physical and Analytical Chemistry, University of Jaen, Campus las lagunillas, s/n. 2307I, Spain, Email ellorent@ujaen.es

Received: July 20, 2017 | Published: July 28, 2017
Abbreviations: FIA, flow-injection analysis; FTO, flowthrough optosensors; MCFIA, multicommutated-flow-injection analysis; PIF, photochemically-induced fluorescence; SIA, sequential injection analysis; SPS, solid-phase spectroscopy

\section{Introduction}

One of the most important aspects of the development of an analytical method is to avoid the use of highly contaminant reagents. Also, the use of low amounts of sample and reagents is important, as fewer wastes are generated. The approach consistent with the development of less hazardous processes for human health and environment is named as 'Green Chemistry', which may be defined as "the use of chemistry techniques and methodologies that reduce or eliminate the use or generation of feedstock's, products, byproducts, solvents, reagents, etc. that are hazardous to human health or the environment". ${ }^{1}$ In Analytical Chemistry, it is important to develop and validate analytical methods that reduce or eliminate the use and release of hazardous substances in all the steps of the analytical process. One of the possible approaches consists in the simplification of sample pre-treatment, using non-toxic chemicals and as low amounts as possible. Another option is the use of automated and miniaturized manifolds. These two approaches agree with the use of low amounts of sample, which allows the decrease of reagents amounts in the sample treatment step. In this review, we will focus on one approach to miniaturize and automate analytical measurements, the use of flowthrough opt sensors.

Spectroscopic flow sensors, flow optosensors, or flow-through optosensors (FTO) are the result of the implementation of solid-phase spectroscopy (SPS) in flow analysis. ${ }^{2}$ SPS consists in the use of an active solid support to pre-concentrate the analyte (or its reaction product) followed by the direct measurement of the analytical signal produced by the analyte while still retained on the solid support. ${ }^{3}$ Early SPS methods provided high selectivity and sensitivity. However, they were carried out in batch mode, which involved tedious and time-consuming procedures. In FTO, the introduction of flow methodologies permits to keep the high sensitivity and selectivity of the original approach but provides automation, improved precision, and rapidity and low reagents consumption. In FTO, the solid support is placed in a flow-through cell, placed inside the detector, just in the detection zone. When the sample solution is inserted in the flow system, the analyte is carried to the flow cell, where it is retained on the solid support. After the analytical signal is measured, the analyte is desorbed from the solid support and the next sample insertion can be made. All the involved steps are usually computer-controlled, hence the improvement in precision.

The most common methodology used in FTO is Flow Injection Analysis (FIA). However, the use of Sequential Injection Analysis (SIA) and multicommutated flow techniques has increased in the last few years, due to their higher degree of automation and lower consumption of reagents. These methodologies will be briefly described in the discussion section. The most common detection techniques in FTO are UV-Visible spectroscopy and fluorescence. Fluorescence detection provides high selectivity and sensitivity. However, when the target compound does not present native fluorescence, other strategies were required. One of them consists in the use of UV-irradiation of the sample solution to produce the photo degradation of the analyte into fluorescent compounds. This strategy is known as photochemically-induced fluorescence (PIF) and can be easily achieved by using a UV lamp. Although the off-line irradiation 
of the sample can be carried out, followed by the measurement of the obtained photoproducts, the implementation of PIF detection in FTO clearly simplifies the procedure, performing the UV irradiation online. This approach will be the main topic of this review.

\section{Discussion}

\section{Flow methodologies in FTO}

In each flow methodology, the flow-cell is placed in the detection zone, and the analyte is retained on the solid support (inside the flow-cell), where the analytical signal is measured. The same principles apply to conventional flow methodologies, i.e. without the implementation of SPS in the flow system. References are provided for each flow methodology for a more in-depth discussion. FIA is based on the injection of the sample into a carrier solution. The injected sample is transported by the carrier towards the detector and, after that, to the waste. The components of an FIA manifold are a peristaltic pump to propel all solutions, plastic tubes to carry the solutions, injection valves (usually 6-way rotary valves) to introduce the solutions in the system, and the detector. The use of FIA provides precise, quick, and versatile analytical methods. However, injection valves are usually human-controlled and wastes generation is higher than in other flow methodologies. ${ }^{4}$

SIA is considered the second generation of flow injection techniques. In SIA manifolds, the peristaltic pumped is replaced by a high-precision syringe pump, whereas the injection valves are replaced by a multi-position valve. Solutions are aspirated by the syringe pump and introduced in a holding coil by the multi-position valve. Then, solutions are pumped towards the detector by the syringe pump. All steps can be computer-controlled, and the versatility of the system is increased, as well as the precision. However, lower sample throughput is obtained in comparison with FIA due to the aspiration/ pumping process that is required. ${ }^{5}$ Finally, multicommutation concept includes the use of commutation devices (usually solenoid valves) to construct dynamic manifolds. As each valve can be individually controlled by appropriate software, these manifolds produce more versatile analytical systems. Solutions can be aspirated or pumped by means of a peristaltic pump, so they are similar to FIA in this aspect. However, they produce low wastes generation and are more precise, as SIA. Hence, they usually present the combined advantages of FIA and SIA, so the use of multicommutated flow methodologies has rapidly increased in the last few years. ${ }^{6}$

\section{PIF manifold}

The different FTO that have been developed using PIF detection could be classified according to two aspects:

\section{a. Number of analytes detected; \\ b. Flow methodology used.}

Most of the FTO was designed for the determination of only one analyte, which is the simplest option. However, the use of an online precolumn filled with an appropriate sorbent can achieve the separation of two compounds. This column can be placed before the UV-lamp, so the analytes are separated before the photo degradation process or after the UV-lamp in order to separate the photoproducts. Taking into account that this separation can be performed no matter the flow methodology that is selected, we will discuss a couple of examples for each flow methodology. As we mentioned before, the manifold will differ depending on the flow methodology that is employed. However, a common aspect is the use of a UV-lamp placed before the detector, so the sample solution can be irradiated online. The photo degradation of the analyte takes place and the photoproduct(s) can then be retained on the solid support, producing the analytical signal.

The early PIF-FTO was designed using FIA. A typical flow configuration is shown in Figure 1. This manifold was used for the determination of imidacloprid in peppers. ${ }^{7}$ Sample solution is injected through injection valve 1 and is pumped through the reaction coil which is wrapping a UV-lamp. The photoproduct is formed online, pumped towards the flow-cell, and retained on the solid support. Once the analytical signal is recorded, the carrier itself desorbs the photoproduct from the solid support. However, an eluting solution (injected through a second rotary valve) is sometimes required to regenerate the solid support, due to a strong interaction between the photoproduct (and other compounds) on the solid support. Figure 1 using the same manifold, but including a pre-column to achieve the separation of the analytes, thiabendazole and metsulfuron methyl were quantified in environmental waters. ${ }^{8}$

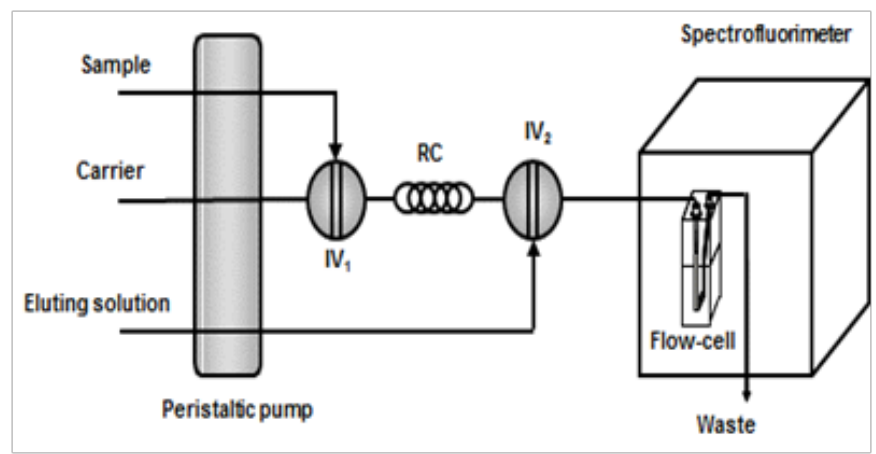

Figue I Typical FIA Manifold for a Mono-Parametric FTOWith PIF Detection. IV, Injection Valve; RC, Reaction Coil.

In this case, thiabendazole was determined by measuring its native fluorescence and metsulfuron methyl by using PIF. To our best knowledge, SIA has not been used for the development of FTO with PIF detection. We will thus discuss a couple of examples of multicommutated procedures for the determination of bioactive compounds in food samples. An example of the simplest multicommutated manifold is shown in Figure 2. This manifold was used for the determination of resveratrol in beer ${ }^{9}$ and piceid in cocoa powder and chocolate. ${ }^{10}$ In both cases, Multicommutated Flow Injection Analysis (MCFIA) was selected. This methodology usually consists in the use of 3-way solenoid valves that present two positions: activated $(\mathrm{ON})$ or deactivated (OFF). Each valve acts as an independent switch, so the ON/OFF position indicates if the flow is directed towards one or another channel. In Figure 2, it can be observed that the carrier solution (controlled by valve 2 ) is continuously flowing through the whole system, including the flow-cell that contains the solid support, whereas sample solution (controlled by valve 2) is recirculated to its corresponding vessel. When both valves are activated, the sample solution is inserted into the system and the analyte suffers on-line UV irradiation when flowing through the reaction coil-coiled around the UV-lamp. Then, sample solution flows through the cell and the photoproduct is retained on the solid support, where it develops its analytical signal. Finally, the solid support is regenerated by the carrier solution, which flows again through the system after the introduction of sample solution has finished. Using a similar approach, the same researchers carried out the simultaneous determination of piceid and resveratrol. ${ }^{11,12}$ In this case, they placed a mini-column filled with $\mathrm{C}_{18}$ 
silica gel after the UV-lamp. The photo degradation products of both compounds were selectively separated on this sorbent, so they could reach the detector in a sequential manner. The approach of separating the photoproducts, although it clearly complicates the analytical system, permits the determination of more than one analyte with a single sample insertion.

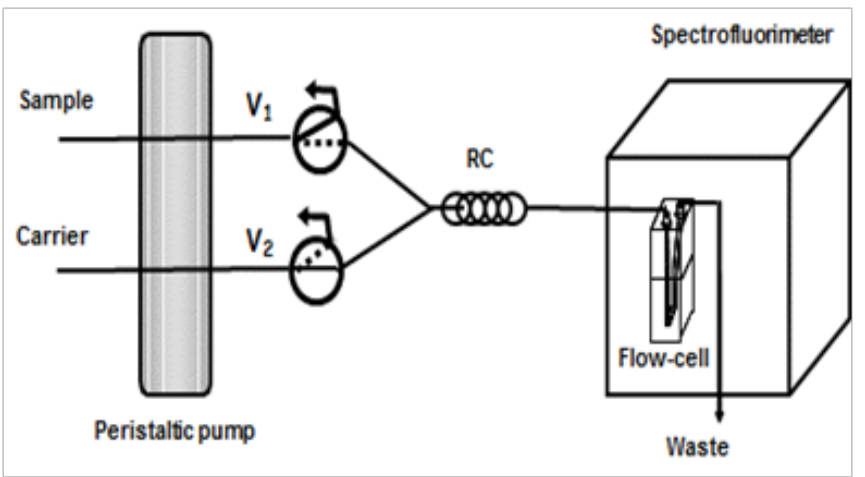

Figure 2 Typical MCFIA Manifold for a Mono-Parametric FTO With PIF Detection. $V_{1}$ and $V_{2}$ are 3-Way Solenoid Valves: Solutions Flow Through The Straight Lines When Valves are OFF and Through de Dotted Lines when Valves are ON. RC, Reaction Coil.

\section{Applications}

In the previous section, we have briefly discussed selected examples of mono-parameter and bi-parameter FTO that used PIF detection. In this section, we present more details of the PIF flow sensors that have been developed to date (Table 1). Most of the developed FTO are monoparametric ones, with few exceptions (Table 1). In all cases, the separation of a binary mixture of analytes was achieved by using a pre-column placed before the detection area. In addition, only FIA and MCFIA were used as flow methodologies, with approximately the same number of FTO developed using each flow technique. ${ }^{13}$ Taking into account that the sample treatment is most of the time the critical step in the development of any analytical method, any type of sampleincluding complex matrices-can be analyzed by FTO. Hence, a wide range of different samples was analyzed, ${ }^{14}$ such as drinking waters, vegetables, milk, pharmaceuticals, etc. In addition, very low detection limits were achieved in most cases, due to the preconcentration step that takes place on the solid support due to the retention of the analyte (similar to the procedure that takes place during a solid-phase extraction (SPE) on a commercial cartridge). ${ }^{11-17}$ Finally, although we have not included sample throughputs in Table 1, they are usually they are usually very high with the possibility of analyzing at least 20-30 samples for hour.

Table I Analytical applications in FTO with PIF detection

\begin{tabular}{|c|c|c|c|c|c|c|}
\hline Analyte & $\begin{array}{l}\text { Solid } \\
\text { Support }\end{array}$ & Matrix & Sample treatment & DL $\left(\mu g \mathbf{L}^{-1}\right)$ & Methodology & Ref \\
\hline Aflatoxin BI & $\mathrm{C}_{18}$ silica gel & Beers & $\begin{array}{l}\text { Degasification; LLE clean-up with toluene } \\
\text { and cyclohexane; SPE with } C_{18}\end{array}$ & 0.029 & MCFIA & 12 \\
\hline \multirow[t]{3}{*}{ Azoxystrobin } & $\mathrm{C}_{18}$ silica gel & Grapes & SLE with $\mathrm{MeOH}+\mathrm{SPE}$ with $\mathrm{C}_{18}$ & 2.4 & MCFIA & 13 \\
\hline & & & SPE with $\mathrm{C}_{18}$ & & & \\
\hline & & Must, wines & & & & \\
\hline Clothianidin & $\begin{array}{l}\text { Sephadex-SP } \\
\text { C- } 25\end{array}$ & $\begin{array}{l}\text { Drinking water; } \\
\text { honey; rice }\end{array}$ & QuEChERS (rice and honey) & 1.5 & MCFIA & 14 \\
\hline Flufenamic acid & $\mathrm{C}_{18}$ silica gel & $\begin{array}{l}\text { Pharmaceuticals; } \\
\text { serum; urine }\end{array}$ & --- & 0.15 & MCFIA & 15 \\
\hline \multirow[t]{3}{*}{ Imidacloprid } & $\mathrm{C}_{18}$ silica gel & Environmental waters & Filtration & 1.8 & FIA & 7 \\
\hline & & & \multicolumn{4}{|c|}{ SLE with acetone + LLE with dichloromethane: light petroleum $(I: I)+$ SPE clean-up with $C_{18}$} \\
\hline & & Peppers & & & & \\
\hline Linuron & $C_{18}$ silica gel & $\begin{array}{l}\text { Environmental and } \\
\text { drinking waters }\end{array}$ & --- & 130 & $\mathrm{FIA}$ & 16 \\
\hline $\begin{array}{l}\text { Metsulfuron } \\
\text { methyl }\end{array}$ & $C_{18}$ silica gel & Environmental waters & Filtration & 0.14 & $\mathrm{FIA}$ & 17 \\
\hline Thiabendazole & $\mathrm{C}_{18}$ silica gel & Environmental waters & Filtration & 2.5 & $\mathrm{FIA}$ & 8 \\
\hline \multicolumn{2}{|c|}{ Metsulfuron methyl } & & & 3.3 & & \\
\hline Piceid & $\begin{array}{l}\text { Sephadex } \\
\text { QAE A-25 }\end{array}$ & $\begin{array}{l}\text { Cocoa powder; } \\
\text { chocolate }\end{array}$ & $\begin{array}{l}\text { Lipid removal; SLE with ethanol: water + } \\
\text { SPE clean-up with } C_{18}\end{array}$ & $\begin{array}{l}0.09-1.4 \mathrm{mg} \\
\mathrm{kg}-\mathrm{I}\end{array}$ & MCFIA & 10 \\
\hline Reserpine & $\mathrm{C}_{18}$ silica gel & $\begin{array}{l}\text { Pharmaceuticals; } \\
\text { serum; urine }\end{array}$ & --- & 0.05 & $\mathrm{FIA}$ & 18 \\
\hline Resveratrol & $\begin{array}{l}\text { Sephadex } \\
\text { QAE A-25 }\end{array}$ & Beer & $\begin{array}{l}\text { LLE clean-up with toluene and } \\
\text { cyclohexane; SPE with } C_{18}\end{array}$ & 1 & MCFIA & 9 \\
\hline Piceid & $\mathrm{C}_{18}$ silica gel & Red and white wine & SPE with $C_{18}$ & 12.6 & MCFIA & 11 \\
\hline Resveratrol & & & & 9.3 & & \\
\hline
\end{tabular}


Table Continued....

\begin{tabular}{|c|c|c|c|c|c|c|}
\hline Analyte & $\begin{array}{l}\text { Solid } \\
\text { Support }\end{array}$ & Matrix & Sample treatment & $D L\left(\mu g L^{-1}\right)$ & Methodology & Ref \\
\hline Sulfonamides & $\begin{array}{l}\text { Sephadex } \\
\text { QAE A-25 }\end{array}$ & Milk; urine & $\begin{array}{l}\text { Clean-up by precipitation with } \mathrm{HCl} \text { and } \\
\mathrm{NaOH} \text {; dilution }\end{array}$ & $2.9-8.1$ & FIA & 19 \\
\hline Thiamethoxam & $\mathrm{C}_{18}$ silica gel & $\begin{array}{l}\text { Lettuce; peppers; } \\
\text { spinach }\end{array}$ & QuEChERS & 3.6 & MCFIA & 20 \\
\hline Thiamine & $C_{18}$ silica gel & $\begin{array}{l}\text { Pharmaceuticals; } \\
\text { serum; urine }\end{array}$ & Filtration & 0.028 & FIA & 21 \\
\hline
\end{tabular}

\section{Conclusion}

The use of FTO is an interesting alternative to other common analytical methods, such as chromatographic ones, although it also presents limitations, such as the impossibility of determining a high number of analytes in a single sample injection. However, it is a lowcost approach for the analysis of a limited number of compounds in selected samples. For the determination of non-fluorescent compounds, PIF detection is a powerful tool. Although the analysis of different compounds in several complex samples has been reported in the last few years, there are still some challenges that have to be addressed in the future. For instance, once the photoproducts are formed, it would be interesting to collect them and try to determine their exact nature. This would provide researchers with useful information for the determination of other analytes of similar nature. In addition, only the most common solid supports- $\mathrm{C}_{18}$ silica gel and Sephadex-have been used. It would be important to carry out experiments with other solid supports, in order to increase the selectivity and sensitivity of the system. Finally, implementing online sample treatments in the flow manifolds would clearly increase the versatility of this analytical methodology. In this regard, the use of other multicommutated techniques, SIA, or Lab-On-Valve may expand the use of PIF sensors. In summary, FTO with PIF detection represent a useful approach, but more innovations are required to widen the range of applications and improve the characteristics of future works.

\section{Acknowledgements}

None.

\section{Conflict of interest}

Author declares that there is no conflict of interest.

\section{References}

1. Anastas PT, Warner JC. Green Chemistry: Theory and Practice. New York, USA; Oxford University Press: 1998. p. 132.

2. Yoshimura K. Implementation of ion-exchanged absorptiometric detection in flow analysis systems. Anal Chem. 1987;59(24):2922-2924.

3. Yoshimura K, Waki H, Ohashi S. Ion-exchanger colorimetry-I Micro determination of chromium, iron, copper and cobalt in water. Talanta. 1976;23(6):449-454.

4. Molina Díaz A, Ruiz Medina A, Fernández De Córdova ML. The potential of flow-through optosensors in pharmaceutical analysis. $J$ Pharm Biomed Anal. 2002;28 (3-4):399-419.

5. Lenehan CE, Barnett NW, Lewis SW. Sequential injection analysis. Analyst. 2002;127(8):997-1020.

6. Llorent Martínez EJ, Ortega Barrales P, Fernández De Córdova ML, et al. Multicommutation in Flow Systems: A Useful Tool for Pharmaceutical and Clinical Analysis. Curr Pharm Anal. 2010;6(1):53-65.
7. López Flores J, Molina Díaz A, Fernández de Córdova ML, et al. Development of a photochemically induced fluorescence-based optosensor for the determination of imidacloprid in peppers and environmental waters. Talanta. 2007;72(3):991-997.

8. López Flores J, Fernández De Córdova ML, Molina Díaz A. Simultaneous flow-injection solid-phase fluorometric determination of thiabendazole and metsulfuron methyl using photochemical derivatization. Anal Sci. 2009;25(5):681-686.

9. Molina García L, Ruiz Medina A, Fernández de Córdova ML. A novel multicommuted fluorimetric optosensor for determination of resveratrol in beer. Talanta. 2011;83(3):850-856.

10. Molina García L, Ruiz Medina A, Fernández de Córdova ML. Automatic optosensing device based on photo-induced fluorescence for determination of piceid in cocoa-containing products. Anal Bioanal Chem. 2011;399(2):965-972.

11. Molina García L, Ruiz Medina A, Fernández De Córdova ML. An automatic optosensing device for the simultaneous determination of resveratrol and piceid in wines. Anal Chim Acta. 2011;689(2):226-233.

12. Molina García L, Fernández de Córdova ML, Ruiz Medina A. Indirect determination of aflatoxin B1 in beer via a multi-commuted optical sensor. Food Addit Contam Part A Chem Anal Control Expo Risk Assess. 2012;29(3):392-402.

13. Flores JL, Díaz AM, Fernández de Córdova ML. Determination of azoxystrobin residues in grapes, musts and wines with a multicommuted flow-through optosensor implemented with photochemically induced fluorescence. Anal Chim Acta. 2007;585(1):185-191.

14. Jiménez López J, Ortega Barrales P, Ruiz Medina A. Determination of clothianidin in food products by using an automated system with photochemically induced fluorescence detection. J Food Comp Anal. 2016;49:49-56.

15. López Flores J, Fernández De Córdova ML, Molina Díaz A. Multicommutated flow-through optosensors implemented with photochemically induced fluorescence: Determination of flufenamic acid. Anal Biochem. 2007;361(2):280-286.

16. Piccirilli GN, Escandar GM, Cañada FC, et al. Flow-through photochemically induced fluorescence optosensor for the determination of linuron. Talanta. 2008;77(2):852-857.

17. Lopez Flores J, Fernandez de Cordova ML, Molina Diaz A. Flowthrough optosensing device implemented with photochemically-induced fluorescence for the rapid and simple screening of metsulfuron methyl in environmental waters. J Environ Monit. 2009;11(5):1080-1085.

18. López Flores J, Fernández de Córdova ML, Molina DíazA. Determination of sub-ppb reserpine by an optosensing device based on photochemically induced fluorescence. Anal Bional Chem. 2007;388(8):1771-1777.

19. López Flores J, Fernández De Córdova ML, Molina Díaz A. Flowthrough optosensor combined with photochemically induced fluorescence for simultaneous determination of binary mixtures of sulfonamides in pharmaceuticals, milk and urine. Anal Chim Acta. 2007;600(2):164-171. 
20. Jiménez López J, Ortega Barrales P, Ruiz Medina A. Development of an semi-automatic and sensitive photochemically induced fluorescence sensor for the determination of thiamethoxam in vegetables. Talanta. 2016;149:149-155.
21. López Flores J, Fernández De Córdova ML, Molina Díaz A Implementation of flow-through solid phase spectroscopic transduction with photochemically induced fluorescence: Determination of thiamine. Anal Chim Acta. 2005;38(27):161-168. 